\title{
Constructing a Dictionary of Human Brain Folding Patterns
}

\author{
Zhong Yi Sun ${ }^{1,3}$, Matthieu Perrot ${ }^{1,3}$, Alan Tucholka ${ }^{1,2,3}$, Denis Rivière ${ }^{1,3}$, \\ and Jean-François Mangin ${ }^{1,3}$ \\ 1 LNAO, Neurospin, I2BM, CEA, Saclay, France \\ zysun@cea.fr \\ http://lnao.fr \\ 2 Parietal, INRIA Saclay, France \\ 3 IFR 49, France \\ http://www.ifr49.org/
}

\begin{abstract}
Brain imaging provides a wealth of information that computers can explore at a massive scale. Categorizing the patterns of the human cortex has been a challenging issue for neuroscience. In this paper, we propose a data mining approach leading to the construction of the first computerized dictionary of cortical folding patterns, from a database of 62 brains. The cortical folds are extracted using BrainVisa open software. The standard sulci are manually identified among the folds. 32 sets of sulci covering the cortex are selected. Clustering techniques are further applied to identify in each set the different patterns observed in the population. After affine global normalization, the geometric distance between sulci of two subjects is calculated using the Iterative Closest Point (ICP) algorithm. The dimension of the resulting distance matrix is reduced using Isomap algorithm. Finally, a dedicated hierarchical clustering algorithm is used to extract out the main patterns. This algorithm provides a score which evaluates the strengths of the patterns found. The score is used to rank the patterns for setting up a dictionary to characterize the variability of cortical anatomy.
\end{abstract}

\section{Introduction}

With the advances in brain imaging techniques such as MR imaging, human brain can be studied non-invasively. We are in an exciting era, when part of the tedious work of mapping the brain conducted by neuroscientists and neurosurgeons can be handled by computers, integrating anatomical, functional and genetic information. However, this work of mapping the brain cannot be accomplished without better understanding of the brain cortical folding anatomy and its variations.

The cortical folding process is relatively consistent, yet a huge variability exists from one individual to another. The primary sulci that appear early on in development, before the 30th week of gestation, are deeper and more stable [12]. The secondary and tertiary folds that form later are more variable from one individual to another 34 . The relationship between the sulcation pattern 
of the cerebral hemispheres and genetics is still controversial despite the efforts of many authors [56]. There are evidences that link folding patterns to brain functionality and pathology 37. However, our knowledge of the functionality around the smaller and less stable folds is far from being certain. It is extremely difficult to study the functional implication of these folding patterns, because of the huge variability that exists across individuals.

The standard approach to overcome this variability is to map the individual brains to an average brain, so that group studies can be carried out using a simple voxel-based strategy [8]. The drawback of this approach is that some spatial information concerning the individual brains is averaged out and lost, which is particularly dramatic when studying the folding patterns. A careful alignment of the main gyral patterns is improving the spatial normalization 101112 but is still hiding the existence of incompatible folding patterns, namely patterns that can not be aligned by a smooth warping. The final average brain is made up of the concatenation of the most frequent local patterns. In places where incompatible patterns occur with similar frequencies, the average brain has unpredictable shapes.

In this paper, we set up a different framework that aims at studying these incompatible patterns. The key point is to split the population into subgroups of brains with similar patterns. This leads to clustering analysis, where we try to find representation clusters that summarize the behaviour of the population. Each of these clusters represents a subgroup of the population. In this paper, the patterns defining these clusters are local but the same framework could be applied to infer global patterns. This framework was already proposed in a previous publication [13], but the shape descriptors used were not sufficient to cover all the complexity of the sulcal patterns. The innovation in this paper over the previous work is a new way to describe the sulcal shapes combining the Iterative Closest Point (ICP) and the Isomap algorithms 914. This innovation leads to a much richer dictionary. The same clustering algorithm as in 1315] is used but we provide a new set of validations dedicated to the new descriptors.

The rest of the paper focuses on our efforts for discovering and mapping the hidden patterns of cortical folding. The goal is to compute the first computerized dictionary of these folding patterns, which is performed from a database of 62 brains. The cortical folds have been extracted using BrainVisa open software. The standard sulci have been manually identified among the folds by human experts. Then these sulci are grouped into 32 different sets with some overlaps. In this paper, we limit the search to these sets. The sulcal sets cover all the regions in the frontal, temporal, parietal and occipital lobes. Some of these regions are defined to address the ambiguities that arise during the labelling process. For example, it is sometimes reported that some brains have two parallel cingulate sulci. Therefore one sulcal set grouping cingulate, intracingulate and paracingulate sulci has been created. The other regions correspond to the gyri which are more intrinsic to the functional organisation than the sulci. For example inferior frontal sulcus and some branches of the Sylvian valley are grouped together to represent Broca's area. Clustering is applied independently to each of these sulcal sets. The method is discussed below and validated using simulated and real datasets. Some results on the real datasets are presented. 


\section{Clustering Algorithm}

The clustering process requires some descriptors of the shape of the sulcal sets. Sophisticated shape descriptors based on 3D moment invariants have been proposed before for this purpose [13. This approach was based on only 12 general descriptors which is not enough to represent all the complexity of the folding patterns: very different shapes can sometimes lead to similar descriptors which is disturbing the clustering. In order to overcome this weakness, we propose in this paper to describe a shape by a vector of distance to a large number of similar shapes. This approach has been proven to be very efficient to compare shapes in large dimension spaces [14. Hence the representation of the sulcal set of one subject is consisting of the distances to the same sulcal set in all the other subjects. Each pairwise distance is computed using the simple ICP algorithm after affine global spatial normalization of the brains $[8$. Note that performing a global normalisation removes non-interesting patterns induced by global differences in brain size. Our ICP implementation is providing the minimal distance obtained whatever the rotation between the two shapes [9].

The input to the clustering analysis are the feature vectors of dimension 62 . The curse of dimensionality is a well-known problem occurring in such situations. Therefore, dimension reduction is applied before clustering using Isomap algorithm. This algorithm has the computational efficiency and global optimality of PCA and MDS, it has also the flexibility to learn a broad class of non-linear manifolds [14]. The input is the distance matrix among the subjects. Linking each point to its $\mathrm{K}$ nearest neighbours, a graph is created that is supposed to describe a low dimensional manifold. Isomap first estimates the geodesic distance between points (here the set of sulci of each subject). The geodesic distance is the length of the shortest path in this graph. It is important to choose an appropriate neighbourhood size. When the neighbourhood is too large, too many "short-circuit" edges would be created; when the neighbourhood is too small, the graph becomes too sparse to approximate the geodesic paths. To our knowledge, there is no consensual general way to choose $\mathrm{K}$ whatever the problem. In this paper, $\mathrm{K}$ has been set to 6 (one tenth of the dataset size). Once the matrix of pairwise geodesic distances has been computed, dimension reduction is achieved using classical MultiDimensional Scaling. This algorithm chooses a N-dimensional configuration minimizing the stress defined by $\Sigma\left(g_{i j}-d_{i j}\right)^{2} / \Sigma d_{i j}^{2}$ where $g_{i j}$ denotes geodesic distances and $d_{i j}$ denotes pairwise distance in the low dimensional space. In the rest of the paper, $\mathrm{N}$ is set to 4 . Further work will be carried out to study the dependence of the final patterns on $\mathrm{K}$ and $\mathrm{N}$.

The clustering algorithm PCBB is applied to the Isomap result. This algorithm has been recently proposed to perform robust detection of tight clusters in a very noisy environment [15. In this specific situation PCBB was shown to perform better than GMM (Gaussian Mixture Models [16]). It provides a score evaluating the significance of the set of detected clusters. The clustering algorithm consists of two steps. In the first step, the number of clusters and their size are estimated automatically. This is performed using a modified hierarchical clustering algorithm, where the p-value of the result is estimated by a parametric 
sampling process. This process is performed many times on bootstrap samples of the original data. The centers of all the clusters detected during the bootstrap are gathered into a new dataset to be clustered in the second step. This two step strategy provides robustness to the clustering. PAM algorithm (Partitioning Around Medoids [17]) is applied to this new dataset to define the final clusters. PAM is an algorithm similar to K-means including estimation of the optimal number of clusters. In our experiments, R cluster toolbox is used 17 . The estimation of the number of clusters performed by PAM in this context has been shown to be reliable [15. The score of the final result is the average of the p-values provided during the first step of PCBB. This score is inversely proportional to the strength of the underlying patterns.

\section{$3 \quad$ Validation and Results}

The motivation for performing dimension reduction with Isomap method is illustrated by Fig 1 , A dataset of $3 * 62$ shapes is generated combining the datasets of three different sulci from our manually labelled database. Dimension reduction of the ICP-based distance matrix is performed with three alternative classical approaches: Isomap, classical MDS and PCA. The result shows that Isomap outperforms the other methods for the separation of sulci.

Next, the performance of the clustering algorithm is evaluated with simulations. Our database of 62 central sulci is used for the generation of simulated datasets. Each of them is composed of 3 simulated tight clusters of 7 sulci plus 41 original central sulci, leading to a total of 62 sulci. For each simulation, three subjects are picked randomly from the original database. Six random variations are generated for each of them. Each variation results from a random transformation applied to the original sulcus. This transformation is an affine transformation endowed with a diagonal of 1 and with 6 random numbers sampled from a Gaussian distribution elsewhere. An example is provided in Fig. 22, 41 additional subjects are picked randomly among the $62-3$ other subjects to
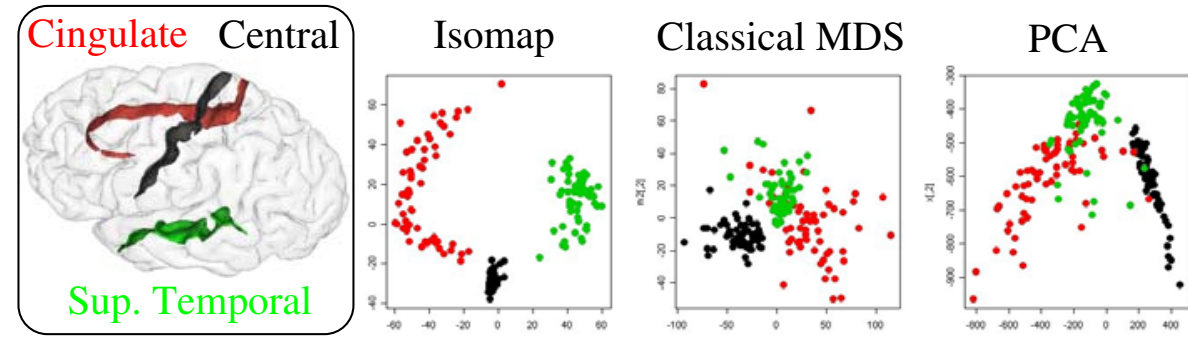

Fig. 1. Comparison of three dimension reduction methods applied on the ICP distance matrix computed for a dataset of $3 \times 62=186$ sulci. The dataset contains 62 different instances of Central (black), Cingulate (red) and Superior Temporal Sulci (green). The dimension is reduced from 186 to 2 . 


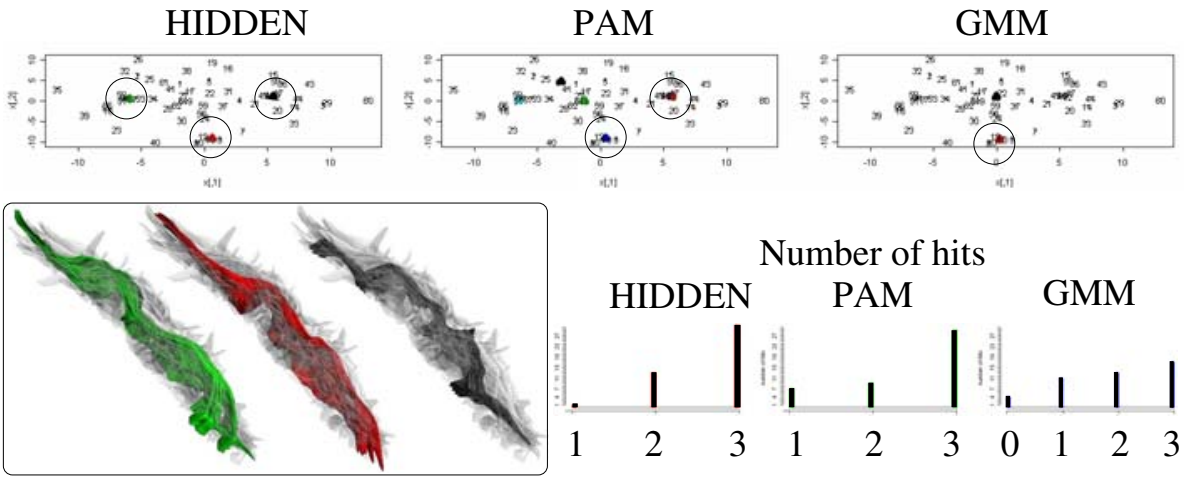

Fig. 2. Validation of the clustering of tight sulcal clusters using 50 simulated datasets. The 62 central sulci of the database are used. Each simulation includes 3 simulated tight clusters of 7 sulci each and 41 original sulci. Each cluster is generated through random deformations of one original sulcus. An example of simulation is provided on the left after ICP-based alignment (0.11 standard deviation, see text). Each color corresponds to one cluster embedded in the 41 transparent original sulci in grey. An example of clustering is provided on top where PCBB detected the three targets, while PAM and GMM missed some. Histograms of the number of hits resulting from 50 simulations are shown on the right.

complete the dataset. Ten different sets of three subjects are picked, and five different standard deviations ranging from 0.11 to 0.15 are used for generating the deformation. A total of 50 simulated datasets are obtained.

For each simulation, the ICP-based distance matrix is computed, Isomap is used for dimension reduction $(\mathrm{K}=6)$. Three different clustering methods are applied: PCBB [15], GMM and PAM. GMM involves first fitting a mixture model by expectation-maximization and computation of posterior probabilities [18. The Bayesian Information Criterion (BIC) provides the number of components. The state-of-the-art Mclust toolbox from $\mathrm{R}$ is used to run GMM [16].

The results are evaluated in terms of the number of simulated clusters found. A detected cluster is considered a hit if the distance from its center to the center of the closest simulated cluster is within the radius of this last cluster. The radius is defined as the median of the distances to the center. Extra clusters found are not penalized since it is possible that the real data contains some clusters. Fig 2 shows a typical result and the performance statistics. PCBB outperforms the two other methods. This is not so surprising since PAM and GMM aim at providing a complete partitioning of the dataset. This goal is not always compatible with the detection of tight clusters.

Clustering was applied to the 32 sulcal sets using PCBB. Group of clusters with scores below 0.05 were collected for the dictionary. 13 sets of the left hemisphere and 12 sets of the right hemisphere passed the threshold. Fig. 3 describes three of the items of our first dictionary. Some of the discovered patterns clearly fit our initial idea of incompatibility. For instance the three patterns of left superior temporal sulcus correspond to a simple long sulcus, a Y-shaped 

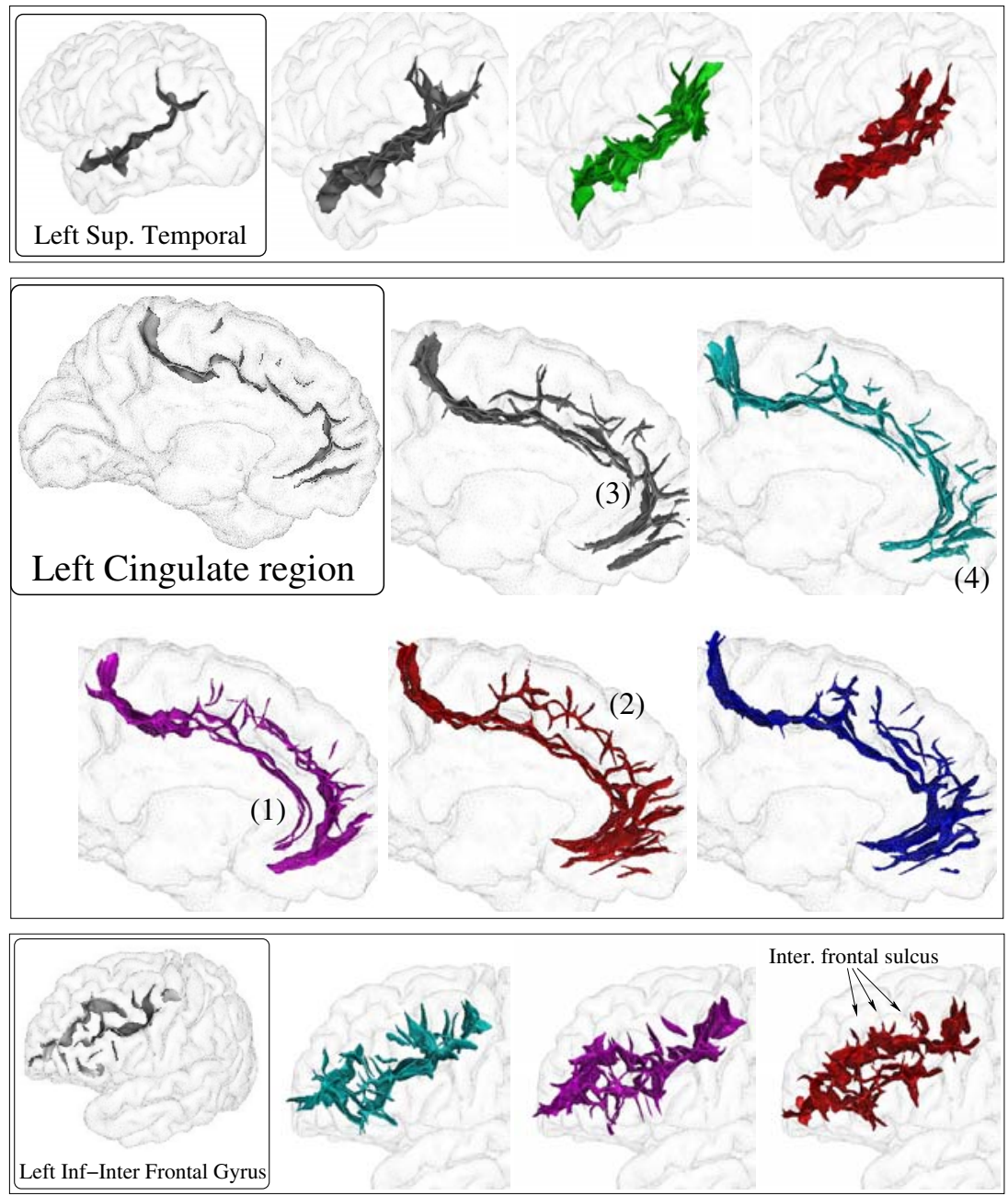

Fig. 3. Three sulcal sets with strong patterns. One example of the sulcal set is shown first. Then for each pattern, three aligned subjects are superimposed in order to highlight the areas of stability making up the patterns. The left superior temporal sulcus exhibits three incompatible configurations of its terminal branches. The left cingulate region is highly variable. The key features are (1) the development of the intracingulate sulcus (long shallow fold at the bottom of violet pattern), (2) the development of the paracingulate sulcus (series of small folds at the top of red pattern), (3) the interruptions of the cingulate sulcus and (4) the shape of the anterior part of the region. The posterior part is relatively stable. The left Inf-Inter frontal gyrus is made up of intermediate precentral and intermediate, marginal, orbitary and inferior frontal sulci. The main difference among the three patterns shown here lies in the different configurations of the intermediate frontal sulcus (small and split: violet, large: red, large and transverse: cyan). 
sulcus, and a split sulcus with two parallel folds in the posterior part. These patterns cannot be aligned easily using spatial normalisation. This implies that if a set of brains can be split into 3 groups based on STS patterns, intra and inter group analysis of functional data could reveal new results. A sophisticated analysis including functional data, diffusion-based tractography and post-mortem histology could help us to define the correct match between the three patterns. Hence, the future of spatial normalisation might lie in a multi-template strategy based on the kind of pattern mapping proposed in this paper.

\section{Conclusion}

The first atlas of the variability of the cerebral sulci was proposed by Ono et al. in 1990 [4. Tedious and patient manual work was required to collect information about a small database of 20 brains. The patterns highlighted in this seminal book were mainly related to sulcus interruptions and sulcus connexions. Taking into account the geometry of the sulci was not possible because this work was performed from flexible moulds of the brains and not from neuroimaging data using computers. Note that the ICP-based distance used for our pattern definition is not based on nonlinear warping but on the smallest distance provided by the rotation group. Therefore our clustering detects not only what was defined in the introduction as incompatible patterns but also compatible patterns that could be aligned by a nonlinear warping. This second kind of patterns could have some interest for morphometric studies.

The first results presented in this paper show that a computerized approach to the mapping of the sulcal patterns could be much more fruitful than any similar manual work. Comparing brains visually is a very difficult task even if computer graphics replaces moulds. Analysing the variability of the folding patterns is often beyond the capacity of the human visual system. On the other hand, we now have access to thousands of MR brain images that could be mined. Some computer vision softwares like BrainVISA can perform the sulcus recognition automatically. These softwares are not perfect. They do not overcome yet the ambiguities resulting from noncompatible patterns. However, applying the method described in this paper to a very large database, we do think that the quality of the automatic sulcus recognition is enough to perform a large scale mapping of the patterns. Much more sulcal sets could be considered.

Pattern analysis adds a wealth of information to our understanding of the variability that exists. For a long time, morphological variability was considered as a difficulty for functional imaging. This led to the design of the spatial normalisation paradigm. The recent understanding that brain morphology could be used to develop early diagnosis in various diseases has raised some new interests in the study of variability 19 . The research on linking the folding patterns with diseases is ongoing. Environmental and genetic factors can both play a role in early brain development, thus affecting the cortical folding process. Hence it is likely that some patterns could be used to define the signature of some developmental diseases. 


\section{References}

1. Régis, J., Mangin, J.F., Ochiai, T., Frouin, V., Rivière, D., Cachia, A., Tamura, M., Samson, Y.: "sulcal root" generic model: a hypothesis to overcome the variability of the human cortex folding patterns. Neurol. Med. Chir. (Tokyo) 45, 1-17 (2005)

2. Lohmann, G., von Cramon, D.Y., Colchester, A.C.: Deep sulcal landmarks provide an organizing framework for human cortical folding. Cerebral Cortex 18(6), 1415$1420(2008)$

3. Welker, W.: Why does cerebral cortex fissure and fold? In: Cerebral Cortex, vol. 8B, pp. 3-136. Plenum Press, New York (1988)

4. Ono, M., Kubik, S., Abernathey, C.D.: Atlas of the cerebral sulci. Thieme (1990)

5. Lohmann, G., von Cramon, D.Y., Steinmetz, H.: Sulcal variability of twins. Cerebral Cortex 9, 754-763 (1999)

6. Le Goualher, G., Argenti, A.M., Duyme, M., et al.: Statistical sulcal shape comparisons: application to the detection of genetic encoding of the central sulcus shape. Neuroimage 11(5), 564-574 (2000)

7. Fischl, B., Rajendran, N., Busa, E., et al.: Cortical folding patterns and predicting cytoarchitecture. Cerebral Cortex 18(8), 1973-1980 (2008)

8. Friston, K., Ashburner, J., Poline, J.B., Frith, C.D., Heather, J.D., Frackowiak, R.S.J.: Spatial realignment and normalisation of images. Human Brain Mapping 2, 165-189 (1995)

9. Besl, P.J., McKay, H.D.: A method for registration of 3D shapes. IEEE Trans. PAMI 14(2), 239-256 (1992)

10. Fischl, B., Sereno, M.I., Tootle, R.B., Dale, A.M.: High-resolution intersubject averaging and a coordinate system for the cortical surface. Human Brain Mapping 8(4), 272-284 (1999)

11. Thompson, P.M., Woods, R.P., Mega, M.S., Toga, A.W.: Mathematical / computational challenges in creating deformable and probabilistic atlases of the human brain. Human Brain Mapping 9(2), 81-92 (2000)

12. Lyttelton, O., Boucher, M., Robbins, S., et al.: An unbiased iterative group registration template for cortical surface analysis. Neuroimage 34(4), 1535-1544 (2007)

13. Sun, Z.Y., Rivière, D., Poupon, F., Régis, J., Mangin, J.-F.: Automatic inference of sulcus patterns using 3D moment invariants. In: Ayache, N., Ourselin, S., Maeder, A. (eds.) MICCAI 2007, Part I. LNCS, vol.4791,pp. 515-522. Springer, Heidelberg (2007)

14. Tenenbaum, J.B., De Silva, V., Langford, J.C.: A global geometric framework for nonlinear dimensionality reduction. Science 290, 2319-2323 (2000)

15. Sun, Z.Y., Rivière, D., Duchesnay, E., Thirion, B., Poupon, F., Mangin, J.F.: Defining cortical sulcus patterns using partial clustering based on bootstrap and bagging. In: 5th Proc. IEEE ISBI, Paris, France, May 2008, pp. 1629-1632 (2008)

16. Fraley, C., Raftery, A.E.: Normal mixture modelling and model-based clustering, Technical Report 504. Department of Statistics, University of Washington (2006)

17. Kaufman, L., Rousseuw, P.J.: In: Finding groups in data. Wiley series in probability and statistics (1990)

18. Duda, R.O., Hart, P.E., Stork, D.G.: In: Pattern Classification. Wiley, Chichester (2000)

19. Ashburner, J., Csernansky, J.G., Davatzikos, C., Fox, N.C., Frisoni, G.B., Thompson, P.M.: Computer-assisted imaging to assess brain structure in healthy and diseased brains. The Lancet Neurology 2, 79-88 (2003) 